\title{
Review
}

\section{White-light-emitting diodes using semiconductor nanocrystals}

\author{
Yanqin Li, Aurora Rizzo, Roberto Cingolani, and Giuseppe Gigli* \\ NNL, National Nanotechnology Laboratory of CNR-INFM, Distretto Tecnologico ISUFI, Engineering of Innovation \\ Department, Lecce University, Via Arnesano Km 5, I-73100 Lecce, Italy
}

Received November 11, 2006; accepted January 19, 2007; published online April 2, 2007

(C) Springer-Verlag 2007

\begin{abstract}
Light-emitting diodes (LEDs) based on colloidal inorganic semiconductor nanocrystals (QDs) represent a completely new technology platform for the development of flat-panel displays and flat-panel lighting systems. Their major advantages are the easy tuning of the saturated color emission across the visible-NIR range and the high chemical and optical stability of the nanocrystal composites. These characteristics open the way to a new class of hybrid devices in which the low cost, flexible technology of organic LEDs is combined with the long operating lifetime of inorganic semiconductor devices. However, so far, few studies have been reported on white-LEDs based on QDs. This review shows recent developments in the general method for the fabrication of stable whiteLEDs comprising QDs with a potentially long lifetime.
\end{abstract}

Key words: Light-emitting diodes (LEDs); white emission; nanocrystals; quantum dots (QDs).

All-organic white-LEDs have been investigated for their potential applications to lighting industry and backlighting applications [1-5]. The main advantages of organic technology for white light generation with respect to other competing technologies, such as GaN based devices [6], stand in the possibility to fabricate large area, eventually flexible, light emitting panels by low cost fabrication techniques, such as evaporation and spin-casting [7-11]. Inorganic LEDs are in fact,

\footnotetext{
* Author for correspondence. E-mail: giuseppe.gigli@ unile.it
}

intrinsically point sources requiring complicated and expensive technological processes when integration in diffused sources is required. To obtain white light from organic systems, different approaches are commonly developed, namely, evaporation or co-evaporation of multilayer structures [7-9], spin-casting of different emitting compounds in a single active layer structure [10, 11], exploitation of exciplex emission [12] and, recently, the synthesis of single white emitting molecules [13]. However, in most of these approaches, the purity of the color emission is strongly affected by the different ageing rate of the active compounds $[7,8]$. In addition, short device lifetimes are obtained due to heating, at the high luminance values required for lighting applications $\left(>1000 \mathrm{~cd} \mathrm{~m}^{-2}\right)$. In this scenario the integration of QDs into LEDs has the potential to overcome most of these problems and it turns out to be a rich field of scientific endeavour [14-19]. The broadly tuneable, saturated color emission performance of QDs are unsurpassed by any class of organic chromophores. Furthermore, the environmental stability of covalently bonded inorganic nanocrystals is expected to increase the device lifetimes at the high luminance/current regimes required for lighting. Moreover, the high quantum yield and narrow band emission of QDs satisfy the technological requirements of flat panel displays and imaging applications. However, despite of all the potentiality of these systems, so far, fewer studies have been reported on white-LEDs based on QDs and the emission 
mechanisms not yet deeply investigated. This review shows recent developments in the general method for the fabrication of stable white-LEDs comprising QDs.

\section{White-LEDs based on blue InGaN chips and QDs}

Two main classes of white-LEDs have been so far reported: multi and single-chip devices. Multichips white-LEDs, constituted by a red-, a green- and a blueemitting chip, show three emission bands and possess a good color rendering index (CRI). However, they are expensive and need a relatively complex external detector and feedback system because each chip degrades at a different rate [20]. Single-chip whiteLEDs will be used as general lighting in the future due to low cost and high luminescence efficiency. Single-chip white-LEDs typically based on yttrium aluminium garnet (YAG) doped with cerium combine low fabrication costs and high efficiency. However, such sources lack a red component giving low color rendering index than that of multichips white-LEDs [21]. To overcome this problem, novel phosphors, for example, organic phosphors with flexibly selected emission color and high luminescence efficiency have been investigated [22-24]. White-LEDs produced by combining a blue InGaN LED chip with phosphors emit an intense bluish white light with optical output levels reaching up to $2 \mathrm{~mW}$ at a drive current of $20 \mathrm{~mA}$ [25]. Nevertheless, the instability and longterm reliability of organic materials are doubtful. On the other hand, colloidal QDs have attracted interest due to their high luminescence efficiency and size-tunable band-gap characteristics [26-30]. Hence, colloidal QDs seem to be suitable as phosphors in white-LEDs. Chen et al. recently demonstrated novel white-LEDs, using white-emitting SA(stearic)/TOPO (trioctylphosphine oxide)-capped ZnSe QDs [31]. The QDs were used as phosphors in a LEDs with a nearUV InGaN chip acting as excitation source. The $\mathrm{ZnSe}$ QDs were mixed with epoxy resin and coated onto the InGaN chip. The device exhibited white emission with a CIE of (0.38 and 0.41), as shown in Fig. 1. The same authors also reported white-LEDs based on blue InGaN chips and core-shell CdSe-ZnSe QDs [32]. Two-band white-LEDs were demonstrated by using blue InGaN chips assembled with yellow-emitting CdSe-ZnSe QDs. Nevertheless, QDs have a narrow spectral band, so the color rendering index of these binary complementary color white-LEDs was low (color rendering index $\mathrm{CRI}=50$ ). This problem has

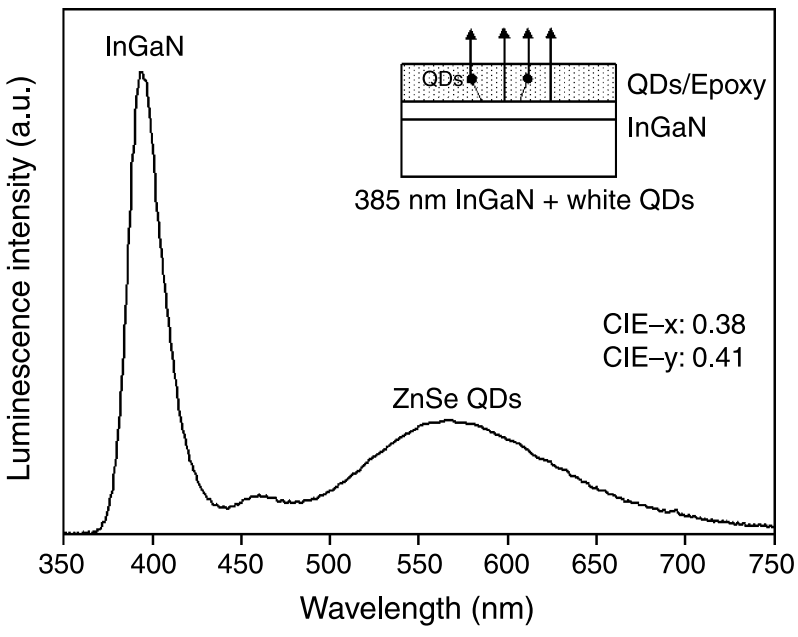

Fig. 1. Luminescence spectrum of a near-UV InGaN/white-emitting QDs white-LEDs (with the copyright permission from AIP)

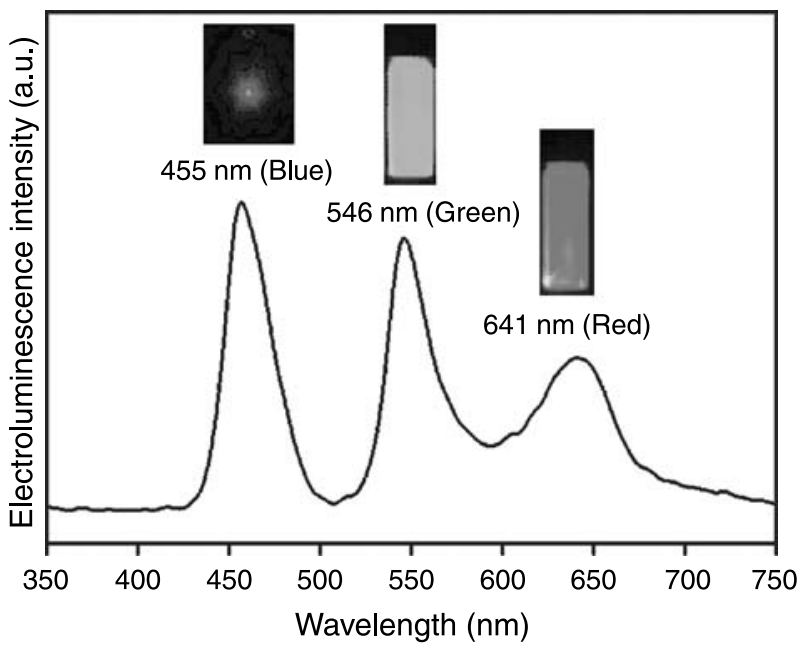

Fig. 2. Three-band white-LEDs combined a blue InGaN chip, green and red-emitting $\mathrm{CdSe}-\mathrm{ZnSe} \mathrm{QDs}$ (with the copyright permission from IEEE Intellectual Property Rights Office)

been overcome by exploiting the broad color tunability of QDs by varying their size. Three-band whiteLEDs, containing a blue InGaN chip, green-emitting QDs, and red-emitting QDs were fabricated with CIE coordinates of $(0.33,0.33)$ and CRI of 91 , as shown in Fig. 2.

\section{White LEDs based on CdSe-ZnS nanocrystals coated on an InGaN-GaN quantum-well}

Recently, the fabrication of phosphor-free, single-chip, all-semiconductor white-LEDs has become an important trend due to the disadvantages of low quantum efficiency, complex synthesis process, patent control and 


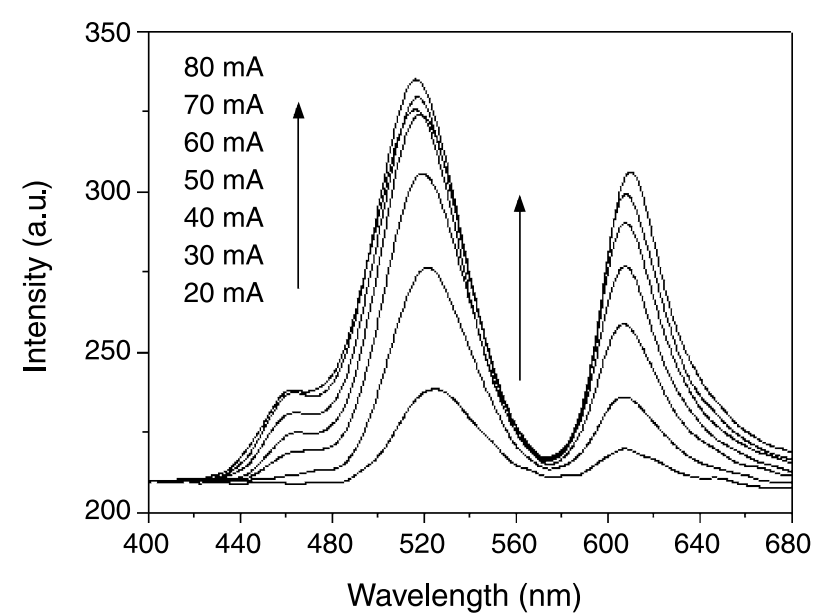

Fig. 3. EL spectra of white-LEDs after CdSe-ZnS nanocrystal coating on a two-wavelength LED at various injection current (with the copyright permission from IEEE Intellectual Property Rights Office)

environmental issue of phosphors for white light generation [33-36]. For this purpose, multiple-wavelength LEDs by stacking InGaN-GaN quantum wells (QWs) of different compositions and/or geometries have been deeply investigated $[33,34]$. In order to obtain white light with a high rendering index by mixing primary colors, currently the major difficulty is the low emission efficiency of the red-emitting InGaN compounds to integrate in the blue- and green-emitting structures. An alternative approach is the use of novel photon down-conversion materials for converting blue photons into red light, with improved stability characteristics with respect to organic phosphors. Recently, it has been proved that the use of $\mathrm{CdSe}-\mathrm{ZnS}$ nanocrystals for such conversion is quite attractive [37-39]. Basically, such QDs can efficiently absorb light in the range from UV through blue and re-emit red light. Its absorption and emission spectra can be easily tuned through controlling its size. Chen et al. first demonstrated the fabrication of a blue/green two-wavelength LED by stacking four different QWs. Then, they show white-LEDs by coating $\mathrm{CdSe}-\mathrm{ZnS}$ nanocrystals on such a two-wavelength LED for converting blue photons into red light [40], as shown in Fig. 3. The suppression of blue light is due to the conversion of blue photons into red light through the $\mathrm{CdSe}-\mathrm{ZnS}$ nanocrystals.

\section{Organic-inorganic hybrid white-LEDs based on polymer/QDs nanocomposites}

To obtain white light, all the three primary colors (red, green and blue) have to be produced simultaneously.
Since it is difficult to obtain all primary emissions from a single molecule, excitation of more than one organic species is often necessary, thus introducing color stability problems. Due to the different degradation rate of the employed organic compounds, the emission color of the device can in fact change with time. Recently, several groups have reported the use of II-VI semiconductor nanocrystals, such as CdSe, in combination with polymers, in the fabrication of LEDs [41-43]. CdSe semiconductor QDs exhibit a size-dependent color variation due to quantum confinement effects, which covers almost the whole visible range. Additionally, the fluorescence efficiency and, in particular the stability of the nanocrystals, can be greatly improved by modifying the particle surface. These characteristics can be merged with peculiar properties of organic materials, such as flexibility and ease of processing, to give rise to a novel class of low cost hybrid white-LEDs with improved lifetime and color stability.

An organic-inorganic hybrid white-LEDs based on polymer/QDs nanocomposites comprising a blue-emitting polymer, PDHFPPV, and CdSe nanoparticles was reported by Kim et al. [44]. Figure 4 shows the EL spectra of the ITO/CdSe:PDHFPPV/Li:Al device measured while applying $10 \mathrm{~V}$. They obtained white emission at bias voltages below $10 \mathrm{~V}$. The partial energy transfer that occurs from the polymer to the QDs enables the QDs emit red light, whose conjunction with the blue light emitted from the polymer,

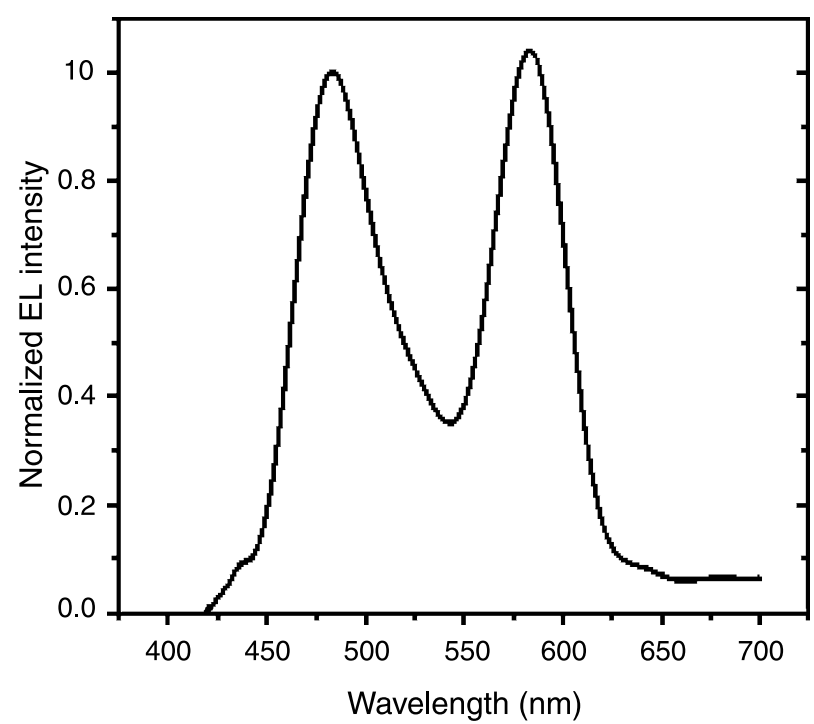

Fig. 4. EL spectrum of the ITO/CdSe:PDHFPPV(6:1 by wt.)/Li: Al device (with the copyright permission from World Scientific Publishing Co., Pte. Ltd.) 
allows the device to generate white emission. The blue-emitting matrix polymer also provides a simple device preparation process due to its high processability. This result suggests that the simple blending method can be employed to obtain polymer-based white-LEDs.

Host-guest systems are typically employed to obtain white light emission by exploiting two mechanisms, namely, Förster energy transfer [45-47] and charge transfer [48-51]. In the Förster mechanism, dipole-dipole coupling results in a nonradiative transfer of the singlet excited-state energy from a donor molecule to an acceptor molecule. In the charge-tansfer mechanism, an excited guest molecule is formed by the sequential transfer of separate hole and electron charges to the guest molecule from different host molecules in the surrounding matrix. All these processes have to be accurately controlled in order to obtain white electroluminescence. In this context we recently demonstrated that a balanced white emission can be obtained in hybrid ternary systems PFHMEH:QDs $/ \mathrm{Alq}_{3}$ when Förster energy transfer in the guest-host system is accomplished by charge transfer from $\mathrm{PFH}-\mathrm{MEH}$ and $\mathrm{Alq}_{3}$ to QDs during the electrical excitation [43]. The chemical structures of the used components and possible energy-transfer, chargetransfer pathways are shown in Fig. 5. Electroluminescence measurements have been carried out on ITO//PEDOT-PSS//PFH-MEH:CdSe/ZnS// $\mathrm{Alq}_{3} / /$ $\mathrm{Ca} / \mathrm{Al}$ structures. In Fig. 6 the EL spectra for different PFH-MEH:CdSe/ZnS concentration ratios are reported. In order to achieve white EL emission the different color components have to be accurately balanced by controlling both the Förster energy transfer and charge transfer mechanisms. To this aim we fabricated devices with different $\mathrm{PFH}-\mathrm{MEH}$ :QDs concentration ra-

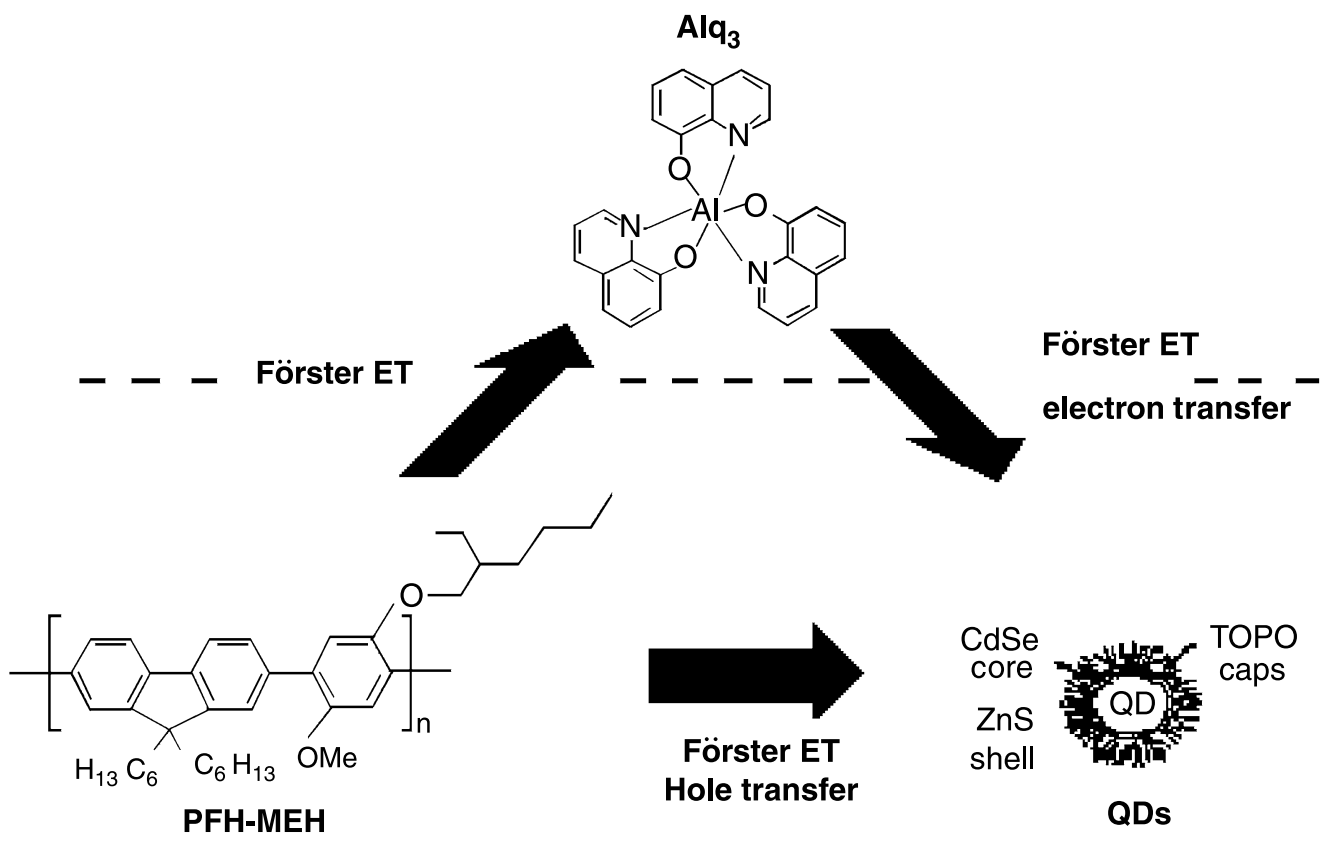

process I

process II

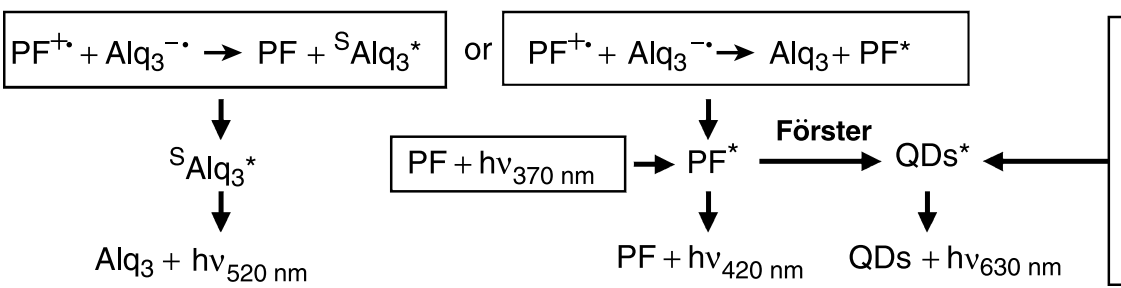

process III

$\mathrm{Alq}_{3}{ }^{-\cdot}+\mathrm{QDs}^{\rightarrow} \rightarrow \mathrm{Alq}_{3}+\mathrm{QDs}^{-\cdot}$
$\mathrm{PF}^{+\cdot}+\mathrm{QDs}^{-\cdot} \rightarrow \mathrm{PF}+\mathrm{QDs}^{*}$
$\mathrm{PF}^{+\cdot}+\mathrm{QDs} \stackrel{\text { or }}{\rightarrow} \mathrm{PF}+\mathrm{QDs}^{+\cdot}$
$\mathrm{Alq}_{3}^{-\cdot}+\mathrm{QDs}^{+\cdot} \rightarrow \mathrm{Alq}_{3}+\mathrm{QDs}^{*}$

Fig. 5. Chemical structures of components and possible pathways leading to emissive states in device ITO//PEDOT-PSS//PFH-MEH (PF): $\mathrm{CdSe} / \mathrm{ZnS} / / \mathrm{Alq}_{3} / / \mathrm{Ca} / \mathrm{Al}$ 


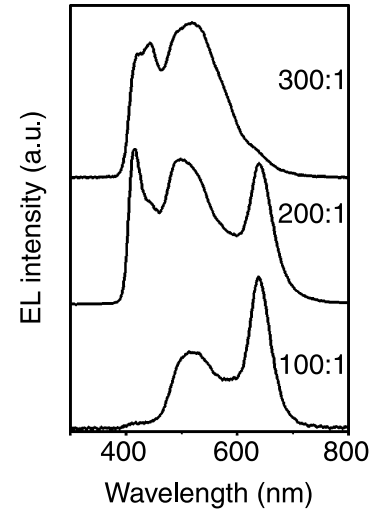

Fig. 6. EL spectra for the device ITO//PEDOT-PSS//PFH$\mathrm{MEH}: \mathrm{CdSe} / \mathrm{ZnS} / / \mathrm{Alq}_{3} / / \mathrm{Ca} / \mathrm{Al}$ with different ratio (PFH-MEH: $\mathrm{CdSe} / \mathrm{ZnS}, \mathrm{c} \%$ )

tios, namely 200:1 and 300:1. At low QDs concentration (300:1), the possible pathway are process I and II. Process I involves the transfer of a hole from a PFH$\mathrm{MEH}$ cation radical $\left(\mathrm{PF}^{+} \cdot\right)$ to a $\mathrm{Alq}_{3}$ anion radical $\left(\mathrm{Alq}_{3}{ }^{-\bullet}\right)$. Process II involves the transfer of an electron from a $\mathrm{Alq}_{3}$ anion radical $\left(\mathrm{Alq}_{3}{ }^{-\bullet}\right)$ to a $\mathrm{PFH}-\mathrm{MEH}$ cation radical $\left(\mathrm{PF}^{+\bullet}\right)$. Both the mechanisms result in excited $\mathrm{Alq}_{3}$ and $\mathrm{PFH}-\mathrm{MEH}$ molecules which can decay radiatively, originating blue and green EL emission. Negligible red emission is instead originated from the low amount of QDs. By increasing the concentration of $\mathrm{CdSe} / \mathrm{ZnS}$ QDs (concentration ratio 200:1), the possible pathway are processes I-III. A relevant additional role is assumed by the following processes: Förster energy transfer to QDs from excited $\mathrm{PFH}-\mathrm{MEH}$ and $\mathrm{Alq}_{3}$ molecules, sequential charge transfer of a hole from PFH-MEH followed by transfer of an electron from $\mathrm{Alq}_{3}$ or charge transfer of an electron from $\mathrm{Alq}_{3}$ followed by transfer of a hole charge from PFH-MEH (process III). Efficient emission at the three primary colors is thus obtained from $\mathrm{PFH}-\mathrm{MEH}, \mathrm{Alq}_{3}$ and QDs with a balanced white spectrum with CIE $(0.30,0.33)$. Maximum External Quantum Efficiency of $0.24 \%$ is measured at $1 \mathrm{~mA} \mathrm{~cm}^{-2}$ and $11 \mathrm{~V}$, to our knowledge the best results so far reported for hybrid white-LEDs based on QDs. In these QDs based white-LEDs, holes are considered to be injected from the ITO electrode through PEDOT:PSS layer into the polymer hole conductor and are eventually transported to the QDs. Similarly, the electrons are considered to be injected from $\mathrm{Ca} / \mathrm{Al}$ cathode into the $\mathrm{Alq}_{3}$ and are eventually transported to the QDs. Since the high electron affinity of QDs the electrons are better confined within the surface PFH-
MEH:QDs $/ \mathrm{Alq}_{3}$, thus enhancing the balance between opposite carriers in the region where more efficient radiative exciton recombination can occur. In particular, charge transfer processes to $\mathrm{CdSe} / \mathrm{ZnS}$ coreshell quantum dots are found to be the key element for well balanced white emission. These results are a first step towards a novel class of hybrid whiteLEDs for lighting applications combining the stability properties of colloidal inorganic nanocrystals with the flexibility and ease of fabrication of organic LED technology.

\section{Bright white-LEDs only from binary or ternary nanocrystals composites}

The key element for a high performance hybrid device in which QDs act as lumophores is the occurrence of efficient exciton recombination in the inorganic nanocrystals. This is usually inhibited by the poor electron conduction of the inorganic species which limits exciton formation $[52,53]$. Therefore low EL efficiency is observed in QDs/polymers blend based devices $[54,55]$. The phase-segregation technique proposed by Coe et al. allows for the fabrication of high efficiency hybrid monochromatic emission devices, but it involves a narrow QDs size distribution to form highcoverage monolayers, which is compatible only with monochromatic emitting QDs-LEDs $[17,56]$. To date, efficient QDs-LEDs that emit white light only from nanocrystals are still a challenge due to the lack of proper fabrication techniques. To obtain white light, all the primary colors (blue $-\mathrm{B}$, green $-\mathrm{G}$ and red $-\mathrm{R}$ ) have to be produced simultaneously. In order to obtain efficient simultaneous emission from different sizes of QDs composites, we recently propose a novel device structures in which exciton formation in the inorganic QDs is not exclusively obtained by direct charge injection but by the accurate control of both the energy/charge transfer mechanisms from the organic host [57]. We demonstrated the first efficient hybrid lightemitting device, with near white emission from chemically and optically stable ternary nanocrystal composites dispersed in an organic matrix. White bright emission is obtained from homogenous blends, without phase segregation between the active ternary QDs composites and the organic matrix, exploiting the energy transfer and charge-trapping properties of the different species. The proposed approach is a new general method for the fabrication of potential long operating lifetime, high efficiency white-LEDs. The 
a

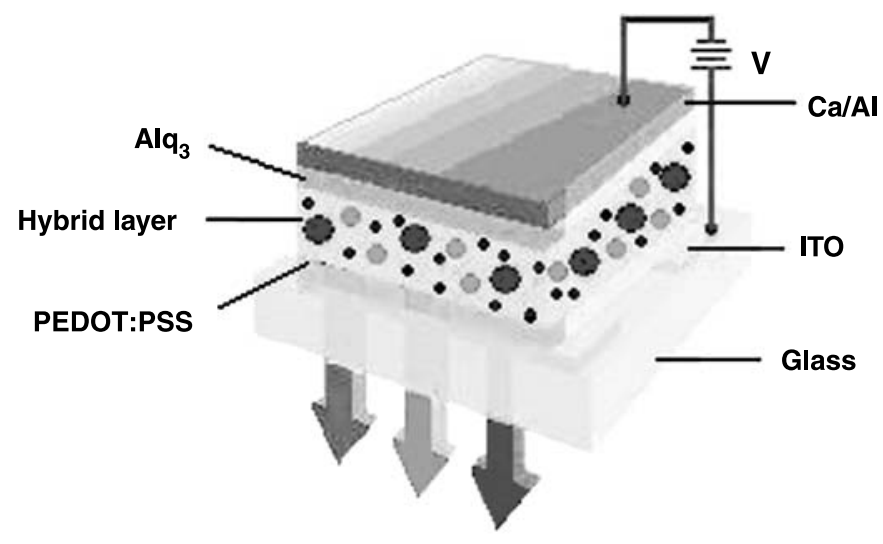

Light output b

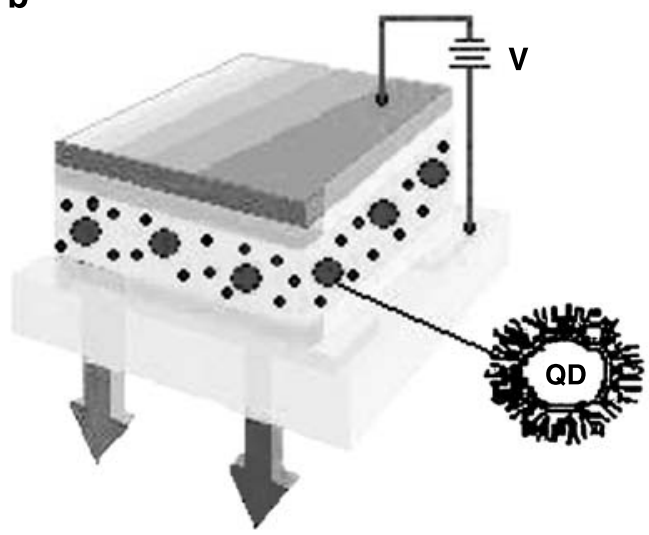

Light output

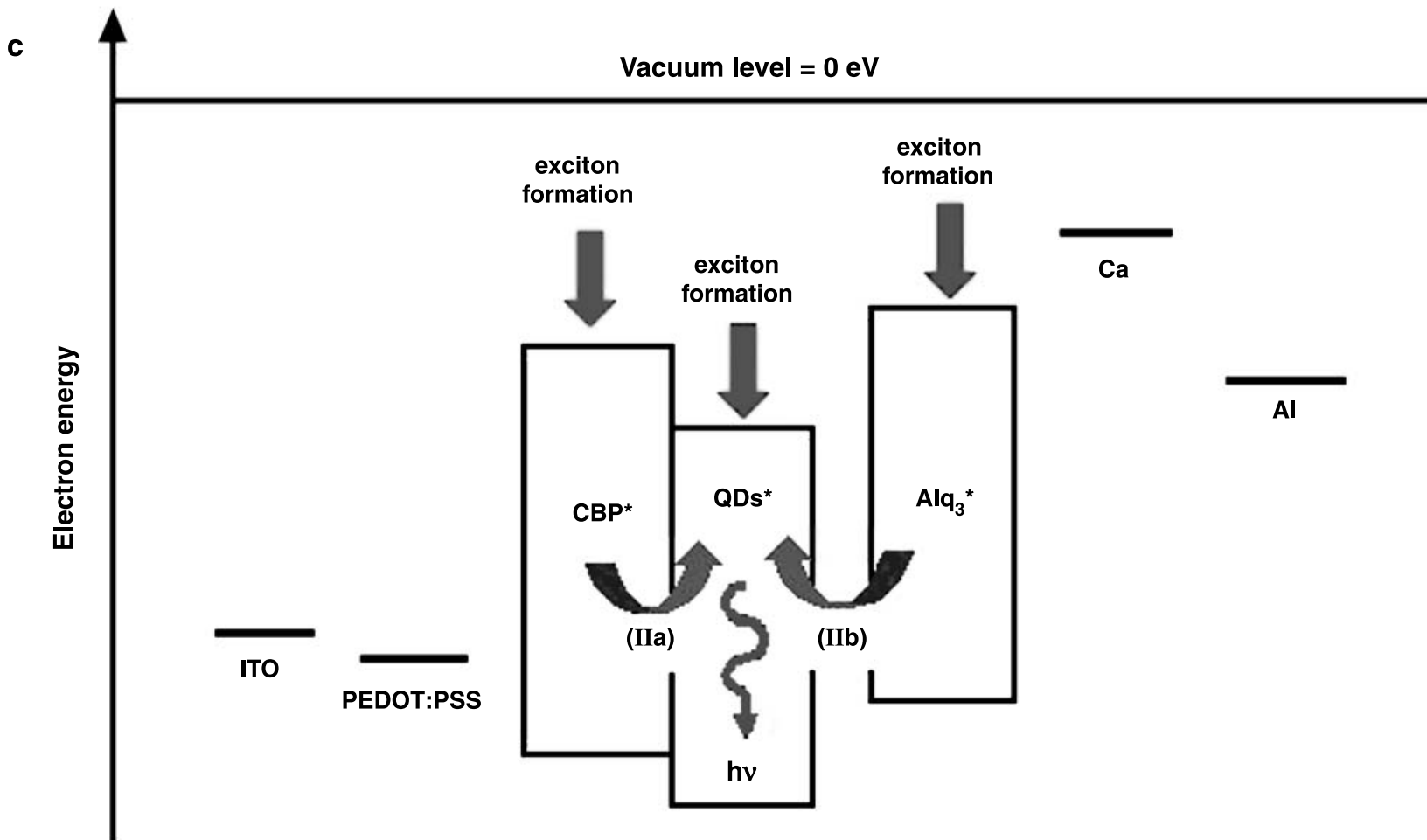

Fig. 7. The structures and simplified energy level diagram of devices and possible exciton creation mechanisms. (a) The structure of the device: ITO//PEDOT:PSS//CBP:QDs(B,G,R)//Alq $/ / / \mathrm{Ca} / \mathrm{Al}$. (b) The structure of the device: ITO//PEDOT:PSS//CBP:QDs(B,R)// $\mathrm{Alq}_{3} / / \mathrm{Ca} / \mathrm{Al}$. (c) Proposed simplified energy level diagram of devices and possible exciton creation mechanisms on the QDs: (I) chargetrapping and (II) Förster energy transfer

structure of the devices and the emission mechanisms are shown in the Fig. 7a and c. As a control, another device structure (Fig. 7b) with binary QDs composites was made. The EL spectrum at $13 \mathrm{~V}$ is shown in Fig. $8 \mathrm{a}$. Spectral peaks at wavelengths of 490,540 and $618 \mathrm{~nm}$ are attributed to the emission of lake blue, green and red quantum dots, respectively, in agreement with the
PL spectra of the isolated QDs in the solid state (see Fig. 8b). A complete quenching of the CBP band is observed in the EL spectrum, resulting in efficient emission at the three primary colors from the QDs, giving rise to a balanced near white overall emission [CIE $(0.32,0.45)]$ at $13 \mathrm{~V}$. Furthermore, the color coordinates do not remarkably change with increasing 

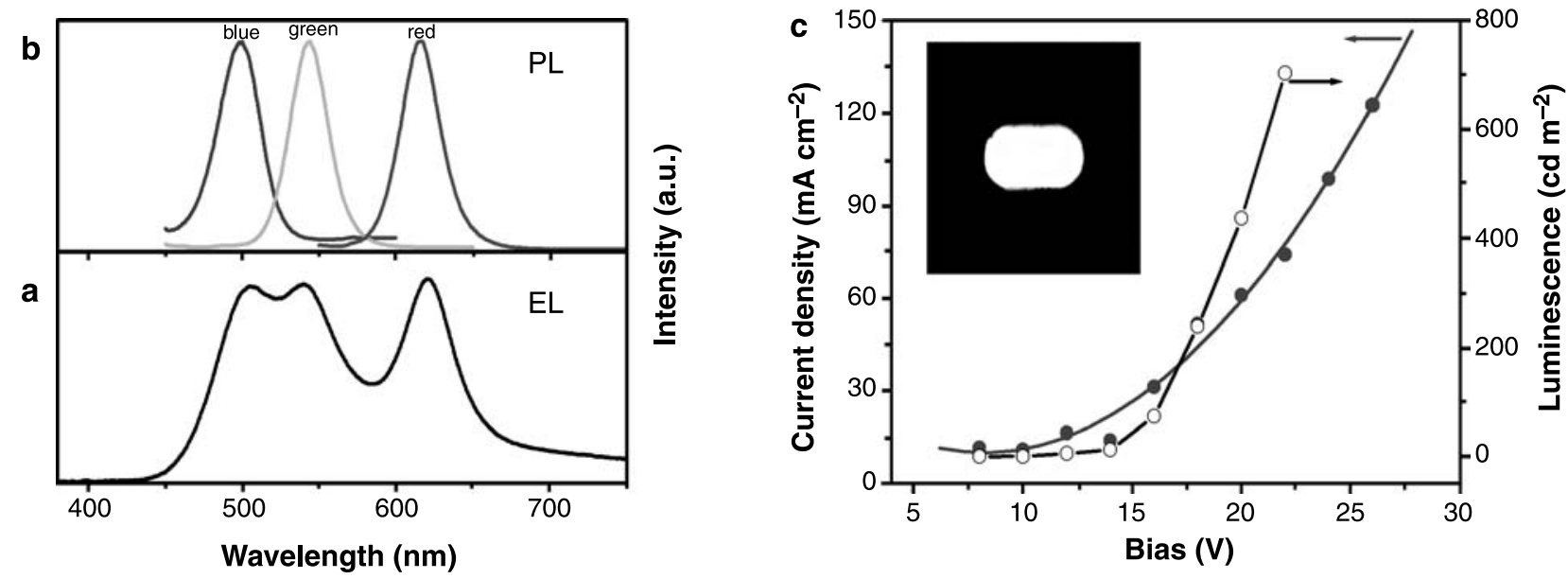

Fig. 8. EL and PL spectra and characteristics of the ternary QDs device. (a) EL spectrum for the device: ITO//PEDOT: PSS//CBP:QDs(B,G,R,c\%=18:2:1)//Alq $/ / / \mathrm{Ca} / \mathrm{Al}$. (b) PL spectra corresponding to isolated lake blue, green and red QDs, which measured in solid state. (c) V-I (dot), V-L (circle) characteristics of the device. Inset: a photo taken from the working device

the operating voltage in the range $10-28 \mathrm{~V}$. This suggests that in the EL spectrum a novel and more efficient transfer mechanism from the host material CBP and the electron transfer layer $\mathrm{Alq}_{3}$, to the lower energy emitting species QDs, occurs. Possible pathways leading to emissive states are shown in Fig. 7c. In our device, holes are injected from the ITO contact through the PEDOT:PSS layer into the CBP host matrix, and are transported towards the QDs. Similarly, electrons are injected from the $\mathrm{Ca} / \mathrm{Al}$ cathode into the $\mathrm{Alq}_{3}$ and are transported to the QDs. Then exciton generation on QDs occurs via two parallel processes: (I) charge-trapping and (II) Förster energy transfer from $\mathrm{CBP}$ and $\mathrm{Alq}_{3}$. In the former process, electrons may be trapped in the QDs owing to the relative energy alignment of the LUMO levels of CBP, $\mathrm{Alq}_{3}$ and QDs, thus, efficient exciton formation on the QDs can occur after recombination with high mobility holes. In the latter process, excitons form on organic molecules $\mathrm{CBP}$ (IIa) and $\mathrm{Alq}_{3}$ (IIb), then undergo Förster energy transfer to the lower-energy QDs sites, where they can recombine radiatively. The different EL and PL spectra evidence that in our hybrid QDs-LEDs chargetrapping is a dominant process for the exciton formation, in agreement with the poor charge conduction properties of $\mathrm{CdSe} / \mathrm{ZnS}$ core-shell QDs [49, 50], and the excellent electron injection/transfer properties of $\mathrm{Alq}_{3}$. In order to achieve white EL the different color emissions from QDs have to be accurately balanced by controlling both the Förster energy transfer and charge-trapping mechanisms, in relation to QDs PL efficiency and concentration. In Fig. 8c volt- age-current (V-I) and voltage-luminance (V-L) characteristics for the device ITO//PEDOT-PSS//CBP: $\mathrm{QDs}(\mathrm{B}, \mathrm{G}, \mathrm{R}) / / \mathrm{Alq}_{3} / / \mathrm{Ca} / \mathrm{Al}$ are reported. The maximum brightness of the device is $1050 \mathrm{~cd} \mathrm{~m}^{-2}$ at $58 \mathrm{~mA} \mathrm{~cm}^{-2}$, which corresponds to a current efficiency of $1.8 \mathrm{~cd} \mathrm{~A}^{-1}$, and a turn-on voltage of $6 \mathrm{~V}$ are measured in air atmosphere. To our knowledge, this is the highest efficiency hybrid device with white emission only from ternary QDs composites, whose luminance satisfies lighting application requirements (i.e. $1000 \mathrm{~cd} \mathrm{~m}^{-2}$ ). As a control, a device structure (Fig. 7b) with only blue and red emitting QDs has been fabricated. EL spectrum demonstrates that the emission is only from QDs composites, and $\mathrm{Alq}_{3}$ functions only as an electron injection and transfer layer. The maximum brightness of the device is $1500 \mathrm{~cd} \mathrm{~m}^{-2}$ at $62 \mathrm{~mA} \mathrm{~cm}^{-2}$, which corresponds to a current efficiency of $2.2 \mathrm{~cd} \mathrm{~A}^{-1}$. As a result, hybrid white emitting LEDs have been fabricated by using three $\mathrm{CdSe} / \mathrm{ZnS}$ QDs of different size blended in a CBP organic matrix. An accurate control and balance of the Förster energy and charge-trapping processes between the different active components, allowed the first high efficiency hybrid device with emission originating only from the inorganic components. The proposed device provides a potential and general method to fabricate long lifetime electroluminescent devices combining the advantages of organic technology in terms of costs and flexibility and of inorganic materials in terms of stability. Color coordinates and light quality can be optimized by a further fine control of the QDs composites. 


\section{Conclusion}

This review shows recent developments in the general method for the fabrication of stable white-LEDs comprising QDs with a potentially long lifetime. As a result, we discussed: white-LEDs produced by combining a blue InGaN LED chip/or an InGaN-GaN two-wavelength QWs LED with colloidal QDs as phosphors; organic-inorganic hybrid white-LEDs wherein a QDs layer, a hole transport layer and/or electron transport layer simultaneously emit light to achieve white emission; QDs with different sizes simultaneously emit light at different wavelength to achieve white emission.

\section{References}

[1] D'Andrade B W, Thompson M E, Forrest S R (2002) Controlling exciton diffusion in multilayer white phosphorescent organic light emitting devices. Adv Mater 14: 147

[2] Zhang X H, Liu M W, Wong O Y, Lee C S, Kwong H L, Lee S T, Wu S K (2003) Blue and white organic electroluminescent devices based on 9,10-bis(2'-naphthyl)anthracene. Chem Phys Lett 369: 478

[3] Cheon K O, Shinar J (2002) Bright white small molecular organic light-emitting devices based on a red-emitting guesthost layer and blue-emitting 4,4'-bis (2,2'-diphenylvinyl)-1, $1^{\prime}$ biphenyl. Appl Phys Lett 81: 1738

[4] Thompson J, Blyth R I R, Mazzeo M, Anni M, Gigli G, Cingolani R (2001) White light emission from blends of blueemitting organic molecules: a general route to the white organic light-emitting diode? Appl Phys Lett 79: 560

[5] Cheng G, Zhao Y, Zhang Y F, Liu S Y, He F, Zhang H Q, Ma Y $\mathrm{G}$ (2004) White organic light-emitting devices using $2,5,2^{\prime}, 5^{\prime}$ tetrakis(4'-biphenylenevinyl)-biphenyl as blue light-emitting layer. Appl Phys Lett 84: 4457

[6] Orton J W, Foxon C T (1998) Group III nitride semiconductors for short wavelength light-emitting devices. Rep Prog Phys 61: 1

[7] Li G, Shinar J (2003) Combinatorial fabrication and studies of bright white organic light-emitting devices based on emission from rubrene-doped 4,4'-bis(2,2'-diphenylvinyl)-1, $1^{\prime}$-biphenyl. Appl Phys Lett 83: 5359

[8] Deshpande R S, Bulović V, Forrest S R (1999) White-lightemitting organic electroluminescent devices based on interlayer sequential energy transfer. Appl Phys Lett 75: 888

[9] Kim C H, Shinar J (2002) Bright small molecular white organic light-emitting devices with two emission zones. Appl Phys Lett 80: 2201

[10] Granström M, Inganäs O (1996) White light emission from a polymer blend light emitting diode. Appl Phys Lett 68: 147

[11] Anni M, Gigli G, Paladini V, Cingolani R, Barbarella G, Favaretto L, Sotgiu G, Zambianchi M (2000) Color engineering by modified oligothiophene blends. Appl Phys Lett 77: 2458

[12] Mazzeo M, Pisignano D, Della Sala F, Thompson J, Blyth R I R, Gigli G, Cingolani R, Sotgiu G, Barbarella G (2003) Organic single-layer white light-emitting diodes by exciplex emission from spin-coated blends of blue-emitting molecules. Appl Phys Lett 82: 334
[13] Mazzeo M, Vitale V, Della Sala F, Anni M, Barbarella G, Favaretto L, Sotgiu G, Cingolani R, Gigli G (2005) Bright white organic light-emitting devices from a single active molecular material. Adv Mater 17: 34

[14] Zhao J L, Zhang J Y, Jiang C Y, Bohnenberger J, Basché T, Mews A (2004) Electroluminescence from isolated CdSe/ZnS quantum dots in multilayered light-emitting diodes. J Appl Phys 96: 3206

[15] Colvin V L, Schlamp M C, Alivisatos A P (1994) Lightemitting diodes made from cadmium selenide nanocrystals and a semiconducting polymer. Nature (London) 370: 354

[16] Lee J, Sundar V C, Heine J R, Bawendi M G, Jensen K F (2000) Full color emission from II-VI semiconductor quantum dot-polymer composites. Adv Mater 12: 1102

[17] Coe S, Woo W K, Bawendi M, Bulović V (2002) Electroluminescence from single monolayers of nanocrystals in molecular organic devices. Nature (London) 420: 800

[18] Coe S, Woo W K, Steckel J S, Bawendi M, Bulović V (2003) Tuning the performance of hybrid organic/inorganic quantum dot light-emitting devices. Org Electron 4: 123

[19] Zhao J L, Bardecker J A, Munro A M, Liu M S, Niu Y H, Ding I K, Luo J D, Chen B Q, Jen A K Y, Ginger D S (2006) Efficient $\mathrm{CdSe} / \mathrm{CdS}$ quantum dot light-emitting diodes using a thermally polymerized hole transport layer. Nano Lett 6: 463

[20] Yam F K, Hassan Z (2005) Innovative advances in LED technology. Microelectron J 36: 129

[21] Sheu J K, Chang S J, Kuo C H, Su Y K, Wu L W, Lin Y C, Lai W C, Tsai J M, Chi G C, Wu R K (2003) White-light emission from near UV InGaN-GaN LED chip precoated with blue/ green/red phosphors. IEEE Photon Technol Lett 15: 18

[22] Hide F, Kozodoy P, Denbaars S P, Heeger A J (1997) White light from InGaN/conjugated polymer hybrid light-emitting diodes. Appl Phys Lett 70: 2664

[23] Zhang C, Heeger A J (1998) Gallium nitride/conjugated polymer hybrid light emitting diodes: performance and lifetime. J Appl Phys 84: 1579

[24] Xiang H F, Yu S C, Che C M, Lai P T (2003) Efficient white and red light emission from GaN/tris-(8-hydroxyquinolato) aluminum/platinum(II) meso-tetrakis(pentafluorophenyl) porphyrin hybrid light-emitting diodes. Appl Phys Lett 83: 1518

[25] Mukai T, Nakamura S (1999) White and UV LEDs. OYO BUTURI 68: 152

[26] Shim M, Guyot-Sionnest P (2000) n-Type colloidal semiconductor nanocrystals. Nature (London) 407: 981

[27] Peng Z A, Peng X G (2001) Formation of high-quality CdTe, $\mathrm{CdSe}$, and CdS nanocrystals using $\mathrm{CdO}$ as precursor. $\mathrm{J} \mathrm{Am}$ Chem Soc 123: 183

[28] Peng X G, Schlamp M C, Kadavanich A V, Alivisatos A P (1997) Epitaxial growth of highly luminescent $\mathrm{CdSe} / \mathrm{CdS}$ core/shell nanocrystals with photostability and electronic accessibility. J Am Chem Soc 119: 7019

[29] Dabbousi B O, Rodriguez-Viejo J, Mikulec F V, Heine J R, Mattoussi H, Ober R, Jensen K F, Bawendi M G (1997) (CdSe) ZnS core-shell quantum dots: synthesis and characterization of a size series of highly luminescent nanocrystallites. J Phys Chem B 101: 9463

[30] Murray C B, Norris D J, Bawendi M G (1993) Synthesis and characterization of nearly monodisperse $\operatorname{CdE}(E=S, S e, T e)$ semiconductor nanocrystallites. J Am Chem Soc 115: 8706

[31] Chen H S, Wang S J J, Lo C J, Chi J Y (2005) White-light emission from organics-capped $\mathrm{ZnSe}$ quantum dots and application in white-light-emitting diodes. Appl Phys Lett 86: 131905

[32] Chen H S, Hsu C K, Hong H Y (2006) InGaN-CdSe-ZnSe quantum dots white LEDs. IEEE Photon Technol Lett 18: 193 
[33] Ozden I, Makarona E, Nurmikko A V, Takeuchi T, Krames M (2001) A dual-wavelength indium gallium nitride quantum well light emitting diode. Appl Phys Lett 79: 2532

[34] Yamada M, Narukawa Y, Mukai T (2002) Phosphor free highluminous-efficiency white light-emitting diodes composed of InGaN multi-quantum well. Jpn J Appl Phys 41: L246

[35] Qi Y D, Liang H, Tang W, Lu Z D, Lau K M (2004) Dual wavelength InGaN/GaN multi-quantum well LEDs grown by metalorganic vapor phase epitaxy. J Cryst Growth 272: 333

[36] Damilano B, Grandjean N, Pernot C, Massies J (2001) Monolithic white light emitting diodes based on $\mathrm{InGaN} / \mathrm{GaN}$ multiple-quantum wells. Jpn J Appl Phys 40: L918

[37] Achermann M, Petruska M A, Kos S, Smith D L, Koleske D D, Klimov V I (2004) Energy-transfer pumping of semiconductor nanocrystals using an epitaxial quantum well. Nature (London) 429: 642

[38] Yeh D M, Huang C F, Chen H S, Tang T Y, Lu C F, Lu Y C, Huang J J, Yang C C, Liu I S, Su W F (2006) Control of the color contrast of a polychromatic light-emitting device with $\mathrm{CdSe}-\mathrm{ZnS}$ nano-crystals on an InGaN-GaN quantum-well structure. IEEE Photon Technol Lett 18: 712

[39] Achermann M, Petruska M A, Koleske D D, Crawford M H, Klimov V I (2006) Nanocrystal-based light-emitting diodes utilizing high-efficiency nonradiative energy transfer for color conversion. Nano Lett 6: 1396

[40] Chen H S, Yeh D M, Lu C F, Huang C F, Shiao W Y, Huang J J, Yang C C, Liu I S, Su W F (2006) White light generation with $\mathrm{CdSe}-\mathrm{ZnS}$ nanocrystals coated on an InGaN-GaN quantumwell blue/green two-wavelength light-emitting diode. IEEE Photon Technol Lett 18: 1430

[41] Yang H, Holloway P H (2003) Electroluminescence from hybrid conjugated polymer-CdSe:Mn/ZnS core/shell nanocrystals devices. J Phys Chem B 107: 9705

[42] Schlamp M C, Peng X G, Alivisatos A P (1997) Improved efficiencies in light emitting diodes made with $\mathrm{CdSe}(\mathrm{CdS})$ core/shell type nanocrystals and a semiconducting polymer. J Appl Phys 82: 5837

[43] Li Y Q, Rizzo A, Mazzeo M, Carbone L, Manna L, Cingolani R, Gigli G (2005) White organic light-emitting devices with $\mathrm{CdSe} / \mathrm{ZnS}$ quantum dots as a red emitter. J Appl Phys 97: 113501
[44] Park J H, Park O O, Kim J K, Yu J W, Kim J Y, Kim Y C (2005) White-electroluminescence device based on polymer/ quantum dot nanocomposites. J Non Opti Phys Mater 14: 481

[45] Förster T (1959) 10th spiers memorial lecture Transfer mechanisms of electronic excitation. Discuss Faraday Soc 27: 7

[46] Turro N J (1978) Modern molecular photochemistry. Benjamin/Cummings, Menlo Park, CA, chap 9

[47] Lakowicz J R (1983) Principles of fluorescence spectroscopy. Plenum, New York, chap 10

[48] Utsugi K, Takano S (1992) Luminescent properties of doped organic EL diodes using naphtalimide derivative. J Electrochem Soc 139: 3610

[49] Suzuki H, Hoshino S (1996) Effects of doping dyes on the electroluminescent characteristics of multilayer organic lightemitting diodes. J Appl Phys 79: 8816

[50] Hikmet R A M, Talapin D V, Weller H (2003) Study of conduction mechanism and electroluminescence in $\mathrm{CdSe} /$ ZnS quantum dot composites. J Appl Phys 93: 3509

[51] Ginger D S, Greenham N C (2000) Charge injection and transport in films of CdSe nanocrystals. J Appl Phys 87: 1361

[52] Leatherdale C A, Kagan C R, Morgan N Y, Empedocles S A, Kastner M A, Bawendi M G (2000) Photoconductivity in CdSe quantum dot solids. Phys Rev B 62: 2669

[53] Greenham N C, Peng X G, Alivisatos A P (1996) Charge separation and transport in conjugated-polymer/semiconductor-nanocrystal composites studied by photoluminescence quenching and photoconductivity. Phys Rev B 54: 17628

[54] Dabbousi B O, Bawendi M G, Onitsuka O, Rubner M F (1995) Electroluminescence from CdSe quantum-dot/polymer composites. Appl Phys Lett 66: 1316

[55] Mattoussi H, Radzilowski L H, Dabbousi B O, Thomas E L, Bawendi M G, Rubner M F (1998) Electroluminescence from heterostructures of poly(phenylenevinylene) and inorganic CdSe nanocrystals. J Appl Phys 83: 7965

[56] Coe S, Steckel J S, Woo W K, Bawendi M G, Bulović V (2005) Large-area ordered quantum-dot monolayers via phase separation during spin-casting. Adv Func Mater 15: 1117

[57] Li Y Q, Rizzo A, Cingolani R, Gigli G (2006) Bright whitelight-emitting device from ternary nanocrystal composites. Adv Mater 18: 2545 